\title{
Autologous EGFR-specific CAR-T-Cells Expressing Anti-PD-1/CTLA-4 Antibodies
}

\author{
National Cancer Institute
}

\section{Source}

National Cancer Institute. Autologous EGFR-specific CAR-T-Cells Expressing Anti-PD-

1/CTLA-4 Antibodies. NCI Thesaurus. Code C150695.

A preparation of autologous T-lymphocytes that have been activated and genetically modified to express immune checkpoint antibodies against the negative immunoregulatory receptors human cell surface receptor programmed cell death protein 1 (PD-1; PDCD1; CD279) and human T-cell receptor cytotoxic T-lymphocyteassociated antigen 4 (CT LA4; CTLA-4), and are transduced with a gene encoding a chimeric antig en receptor (CAR) specific for the human tumor-associated antigen (TAA) epidermal growth factor receptor (EGFR), with potential immunomodulating and antineoplastic activities. After isolation, activation, transduction, expansion in culture and reintroduction into the patient, the T-cells in the autologous EGFR specific CAR-T-cells expressing anti-PD-1/CT LA4 antibodies specifically target and kill EGFR-expressing tumor cells. The anti-PD-1 antibody secreted from the CAR-T cells binds to PD-1 expressed on T-cells and prevents the interaction of PD-1 with its ligand programmed cell death 1 ligand 1 (PD-L1, PD-1L1; CD274) expressed on cancer cells, which prevents PD-1mediated signaling and T-cell exhaustion. The anti-CT LA4 expressed by the CAR-T cells targets and binds to CT LA4 expressed on T-cells, and inhibits the CT LA4-mediated downregulation of T-cell activation. Both antibodies enhance T-cell activation, improve immunosuppression in the tumor microenvironment (TME) and improve the T-cell mediated immune response against and toxicity in EGFR-expressing tumor cells. Both PD-1 and CT LA-4 negatively regulate T-cell activation and proliferation, and play a key role in immunosuppression within the TME. EGFR, a receptor tyrosine kinase that is overexpressed in a variety of cancer cell types, plays a key role in tumor cell proliferation. 